Portland State University

PDXScholar

Electrical and Computer Engineering Faculty

Publications and Presentations

8-1-2003

\title{
Spontaneous Coherent Pulsations in 3.39-m He-Ne Standing-Wave Laser Oscillators
}

Pitak Chenkosol

Lee W. Casperson

Portland State University

Follow this and additional works at: https://pdxscholar.library.pdx.edu/ece_fac

Part of the Electrical and Computer Engineering Commons

Let us know how access to this document benefits you.

\section{Citation Details}

Pitak Chenkosol and Lee W. Casperson, "Spontaneous coherent pulsations in 3.39-m He-Ne standingwave laser oscillators," J. Opt. Soc. Am. B 20, 2539-2547 (2003).

This Article is brought to you for free and open access. It has been accepted for inclusion in Electrical and Computer Engineering Faculty Publications and Presentations by an authorized administrator of PDXScholar. Please contact us if we can make this document more accessible: pdxscholar@pdx.edu. 


\title{
Spontaneous coherent pulsations in 3.39- $\mu \mathrm{m}$ He-Ne standing-wave laser oscillators
}

\author{
Pitak Chenkosol and Lee W. Casperson \\ Departments of Electrical and Computer Engineering and of Physics, Portland State University, P.O. Box 751, \\ Portland, Oregon 97207-0751
}

Received March 20, 2003; revised manuscript received August 1, 2003; accepted August 7, 2003

\begin{abstract}
Spontaneous pulsations similar to the type discovered in $3.51-\mu \mathrm{m}$ Xe laser oscillators were reported several years ago in 3.39- $\mu \mathrm{m} \mathrm{He}-\mathrm{Ne}$ lasers, but no numerical interpretation of the $\mathrm{He}-\mathrm{Ne}$ data has been given. The model for the Xe laser instability is adapted here to the $\mathrm{He}-\mathrm{Ne}$ system, and the results include direct comparisons between theoretical calculations and published experimental data. Good agreement is obtained for the instability threshold, the pulsation frequencies, and many other features; and unmeasured but potentially useful pulsation characteristics are also readily obtained from the model. (C) 2003 Optical Society of America OCIS codes: 270.3430, 270.3100, 020.1670, 140.3460.
\end{abstract}

\section{INTRODUCTION}

Since the invention of the laser, many lasers have been discovered that, with time-independent pumping and cavity loss rates, sometimes produce their outputs in the form of infinite trains of undamped pulsations. With some lasers, these pulsations may be highly periodic, so they cannot be interpreted as resulting from noise-driven fluctuations in a stable system. Many explanations have been proposed for the observed laser-instability phenomena. In some cases, the instability mechanism is well understood, while in others substantial doubt remains.

The simplest laser may be imagined to consist of a uniformly distributed low-density set of atoms interacting with a plane-wave single-frequency cavity field by means of the standard processes of stimulated emission, absorption, and spontaneous emission. The semiclassical Maxwell-Schrödinger equations governing such a system already include the possibility of a destabilizing effect, and this intrinsic instability mechanism is thought to provide an essentially complete explanation for the spontaneous pulsations observed in Xe lasers. ${ }^{1,2}$ On the other hand, it has been uncertain whether this mechanism contributes significantly to the spontaneous pulsations observed in any other class of laser. The light-matter interactions in most lasers can be described adequately by means of simpler rate equations, and in the rate-equation models, spontaneous pulsations require the presence of some supplementary process. Proposed instabilitycausing mechanisms have included saturable absorption within or at the ends or sides of the laser medium or elsewhere in the laser cavity, nonlinear interactions among multiple transverse or longitudinal cavity modes, focusing or defocusing effects caused by nonlinear gain, refraction, or reflection, more complex energy-level structure, and other possibilities. Several reviews of spontaneouspulsation instabilities are available. ${ }^{3-9}$ Although many of the basic concepts in this subject have been well established for many years, research continues. Recent devel- opments have included pulsations in fiber lasers ${ }^{10}$ and new multilevel effects. ${ }^{11,12}$

The theoretical model underlying the spontaneouspulsation effect in Xe lasers predicts similar behavior for a range of high-gain inhomogeneously broadened lasers, and one might have expected that other similar spontaneously pulsing systems would have been reported. A pulsation instability has in fact been observed in the $3.39-\mu \mathrm{m}$ $\mathrm{He}-\mathrm{Ne}$ laser, and experimental data have been published. ${ }^{13,14}$ However, after several years, these data still have not been interpreted in terms of a numerical model, and no other comparable laser system has been shown to exhibit similar behavior. In this circumstance one can wonder whether the He-Ne instability is actually due to the same mechanism as that accepted for Xe lasers or whether the Xe mechanism applies to any other lasers. Further experimental examples with rigorous modeling could answer these questions and reinforce the basic semiclassical instability interpretation.

A major purpose of this study is to systematically compare the available spontaneous-pulsation data for $3.39-\mu \mathrm{m} \mathrm{He}-\mathrm{Ne}$ lasers with a model of the type that was previously successful for Xe lasers. Good agreement is obtained for both the threshold for instability and the corresponding pulsation frequency. Other previously reported features, such as the complex structures of the homodyne and heterodyne spectra as the threshold parameter or the cavity detuning is varied, the narrow range of pulsations around the line center, the anomalous modepulling, and the varying depth and width of the Lamb dip, are also in agreement with the theory.

A normalized form of a general semiclassical singlemode standing-wave laser model is reviewed in Section 2. A set of parameters for $3.39-\mu \mathrm{m} \mathrm{He}-\mathrm{Ne}$ lasers is developed and discussed in Section 3. Detailed numerical calculations of the pulsation phenomena in $\mathrm{He}-\mathrm{Ne}$ lasers are carried out, and comparisons of these results with published experimental data are presented in Section 4. 


\section{MODEL}

The spontaneous pulsations in xenon lasers have been interpreted in terms of a fundamental instability in the semiclassical Maxwell-Schrödinger model for a gas-laser oscillator. The Maxwell-Schrödinger approach was introduced by Lamb within a few years of the invention of the laser, ${ }^{15}$ and with extensions this model was found to provide good agreement with the pulsation behavior of $\mathrm{Xe}$ lasers. While the self-pulsing phenomenon in Xe lasers has been demonstrated experimentally for both the unidirectional ring and standing-wave configurations, most theoretical studies have been directed toward the unidirectional ring oscillators. ${ }^{16,17}$ This is because the spatial distribution of the electric field in a ring-type cavity allows a description in terms of simpler semiclassical laser equations compared with those of the standing-wave cavity.

Most practical lasers are, however, of the standingwave type, and a rigorous semiclassical model for standing-wave laser oscillators has also been derived. ${ }^{18}$ To help in understanding this model and the parameters that are needed for its application, the final normalized form from a previous Xe laser study is reproduced here. Thus the fundamental differential equations of the model can be written as follows ${ }^{19}$ :

$$
\begin{aligned}
\frac{\partial}{\partial t} P_{r, k}(V, t)= & -\gamma\left\{[1+(2 k-1) i V] P_{r, k}(V, t)\right. \\
& +y P_{i, k}(V, t)+i A_{i}(t) \\
& \times\left[\epsilon \frac{\exp \left(-\epsilon^{2} V^{2}\right)}{\pi^{1 / 2}}\left\{E_{k}(t)-E_{k+1}(t)\right\}\right. \\
& \left.\left.+D_{k}^{\prime}(V, t)-D_{k+1}^{\prime}(V, t)\right]\right\},
\end{aligned}
$$$$
\frac{\partial}{\partial t} P_{i, k}(V, t)=-\gamma\left\{[1+(2 k-1) i V] P_{i, k}(V, t)\right.
$$$$
-y P_{r, k}(V, t)-i A_{r}(t)
$$$$
\times\left[\epsilon \frac{\exp \left(-\epsilon^{2} V^{2}\right)}{\pi^{1 / 2}}\left\{E_{k}(t)-E_{k+1}(t)\right\}\right.
$$$$
\left.\left.+D_{k}^{\prime}(V, t)-D_{k+1}^{\prime}(V, t)\right]\right\}
$$$$
\frac{\partial}{\partial t} D_{k}^{\prime}(V, t)=-\left[h_{1}+(2 k-2) i \gamma V\right] D_{k}^{\prime}(V, t)
$$$$
-h_{2} M_{k}^{\prime}(V, t)-(2 k
$$$$
-2) i \gamma V E_{k}(t) \epsilon \frac{\exp \left(-\epsilon^{2} V^{2}\right)}{\pi^{1 / 2}}
$$$$
-i \gamma_{1}\left[A_{r}(t) P_{i, k-1}(V, t)\right.
$$$$
\text { - } A_{i}(t) P_{r, k-1}(V, t)-A_{r}(t) P_{i, k}(V, t)
$$$$
\left.+A_{i}(t) P_{r, k}(V, t)\right] \text {, }
$$

$$
\begin{aligned}
& \frac{\partial}{\partial t} M_{k}^{\prime}(V, t)=-\left[h_{3}+(2 k-2) i \gamma V\right] M_{k}^{\prime}(V, t) \\
& -h_{4} D_{k}^{\prime}(V, t)-(2 k \\
& -2) i \gamma V F_{k}(t) \epsilon \frac{\exp \left(-\epsilon^{2} V^{2}\right)}{\pi^{1 / 2}}, \\
& \frac{\mathrm{d}}{\mathrm{d} t} E_{k}(t)=\left[L_{a}(t)-L_{b}(t)\right] \delta_{k 1}-h_{5} E_{k}(t) \\
& -h_{6}(t) F_{k}(t) \\
& +\frac{\Gamma_{a}}{2} \int_{-\infty}^{\infty}\left[M_{k}^{\prime}\left(V^{\prime}, t\right)+D_{k}^{\prime}\left(V^{\prime}, t\right)\right] \mathrm{d} V^{\prime} \\
& -\frac{\Gamma_{b}}{2} \int_{-\infty}^{\infty}\left[M_{k}^{\prime}\left(V^{\prime}, t\right)\right. \\
& \left.-D_{k}^{\prime}\left(V^{\prime}, t\right)\right] \mathrm{d} V^{\prime} \\
& \frac{\mathrm{d}}{\mathrm{d} t} F_{k}(t)=\left[L_{a}(t)+L_{b}(t)\right] \delta_{k 1}-h_{7} F_{k}(t) \\
& -h_{8}(t) E_{k}(t) \\
& +\frac{\Gamma_{a}}{2} \int_{-\infty}^{\infty}\left[M_{k}^{\prime}\left(V^{\prime}, t\right)+D_{k}^{\prime}\left(V^{\prime}, t\right)\right] \mathrm{d} V^{\prime} \\
& +\frac{\Gamma_{b}}{2} \int_{-\infty}^{\infty}\left[M_{k}^{\prime}\left(V^{\prime}, t\right)\right. \\
& \left.-D_{k}^{\prime}\left(V^{\prime}, t\right)\right] \mathrm{d} V^{\prime} \\
& \frac{\mathrm{d}}{\mathrm{d} t} A_{r}(t)=-\frac{1}{2 t_{c}}\left[A_{r}(t)+\delta\left(y-y_{0}\right) A_{i}(t)\right. \\
& \left.-\int_{-\infty}^{\infty} P_{i, 1 i}(V, t) \mathrm{d} V\right] \text {, } \\
& \frac{\mathrm{d}}{\mathrm{d} t} A_{i}(t)=-\frac{1}{2 t_{c}}\left[A_{i}(t)-\delta\left(y-y_{0}\right) A_{r}(t)\right. \\
& \left.+\int_{-\infty}^{\infty} P_{r, 1 i}(V, t) \mathrm{d} V\right] \text {. }
\end{aligned}
$$

The dependent variables in Eqs. (1)-(8) include the $k$ th spatial harmonic of the real part $P_{r, k}(V, t)$ and the imaginary part $P_{i, k}(V, t)$ of the polarization, the $k$ th spatial harmonic of the broad Gaussian part of the difference between the populations of the upper and lower laser levels $E_{k}(t)$ and their sum $F_{k}(t)$, and the narrow part that reflects the localized field-caused distortion of the population difference $D_{k}^{\prime}(V, t)$ and population $\operatorname{sum} M_{k}^{\prime}(V, t)$. These variables are related to the $k$ th harmonic of the total population difference $D_{k}(V, t)$ and population sum $M_{k}(V, t)$ by $^{19}$

$$
D_{k}(V, t)=\frac{\epsilon E_{k}(t)}{\pi^{1 / 2}} \exp \left(-\epsilon^{2} V^{2}\right)+D_{k}^{\prime}(V, t),
$$




$$
M_{k}(V, t)=\frac{\epsilon F_{k}(t)}{\pi^{1 / 2}} \exp \left(-\epsilon^{2} V^{2}\right)+M_{k}^{\prime}(V, t),
$$

The variables $A_{r}(t)$ and $A_{i}(t)$ are the real and imaginary parts of the complex amplitudes of the electric field. The spatially independent total pumping rates of the upper and lower laser levels are represented by $L_{a}(t)$ and $L_{b}(t)$, respectively. The decay rates include the total decay rate from the upper laser level $\gamma_{a}$, the total decay rate from the lower laser level $\gamma_{b}$, the rate of direct decay from the upper laser level to the lower laser level $\gamma_{a b}$, the polarization decay rate $\gamma$, and the electric-field decay rate $\left(2 t_{c}\right)^{-1}$, where $t_{c}$ is the cavity lifetime. The coefficients $\Gamma_{a}$ and $\Gamma_{b}$ are the total rates at which the atoms in levels $a$ and $b$ undergo strong velocity-changing collisions, respectively. The effect of these collisions is to distribute the final velocities of the atoms randomly across the Doppler gain profile. The velocity integrals in Eqs. (5) and (6) represent the collision-caused additions of atoms to each velocity class. On the other hand, the collisioncaused losses of atoms from each velocity class can be accounted for by replacement of the spontaneous-emission decay rates $\gamma_{a}$ and $\gamma_{b}$ with new effective values $\gamma_{a}^{\prime}=\gamma_{a}$ $+\Gamma_{a}$ and $\gamma_{b}^{\prime}=\gamma_{b}+\Gamma_{b}$. These decay rates are represented in the model by a set of hybrid decay rates, ${ }^{19}$

$$
\begin{aligned}
& h_{1}=\left(\gamma_{a}^{\prime}+\gamma_{a b}+\gamma_{b}^{\prime}\right) / 2, \\
& h_{2}=\left(\gamma_{a}^{\prime}+\gamma_{a b}-\gamma_{b}^{\prime}\right) / 2, \\
& h_{3}=\left(\gamma_{a}^{\prime}-\gamma_{a b}+\gamma_{b}^{\prime}\right) / 2, \\
& h_{4}=\left(\gamma_{a}^{\prime}-\gamma_{a b}-\gamma_{b}^{\prime}\right) / 2, \\
& h_{5}=h_{1}-\Gamma_{a} / 2-\Gamma_{b} / 2=\left(\gamma_{a}+\gamma_{a b}+\gamma_{b}\right) / 2, \\
& h_{6}=h_{2}-\Gamma_{a} / 2+\Gamma_{b} / 2=\left(\gamma_{a}+\gamma_{a b}-\gamma_{b}\right) / 2, \\
& h_{7}=h_{3}-\Gamma_{a} / 2-\Gamma_{b} / 2=\left(\gamma_{a}-\gamma_{a b}+\gamma_{b}\right) / 2, \\
& h_{8}=h_{4}-\Gamma_{a} / 2+\Gamma_{b} / 2=\left(\gamma_{a}-\gamma_{a b}-\gamma_{b}\right) / 2, \\
& \gamma_{1}=2 \gamma_{a}^{\prime} \gamma_{b}^{\prime} /\left(\gamma_{a}^{\prime}-\gamma_{a b}+\gamma_{b}^{\prime}\right) .
\end{aligned}
$$

The parameter $y=\left(\omega-\omega_{0}\right) / \gamma$ in Eqs. (1), (2), (7), and (8) represents the normalized difference between an arbitrarily selected lasing frequency $\omega$ and the atomic linecenter frequency $\omega_{0}$, and $y_{0}$ represents the corresponding parameter when the dispersion effects are neglected. The normalized axial component of velocity is $V$. The parameter $\epsilon=\left(\Delta v_{h} / \Delta v_{D}\right)(\ln 2)^{1 / 2}$ is the so-called natural damping ratio, which measures the relative magnitude of the homogeneous and inhomogeneous linewidths, and the parameter $\delta=2 \gamma t_{c}$ measures the ratio of the polarization and the electric-field decay rates. Equations (1)-(6) follow from the semiclassical equations that govern the four elements of the density matrix for the two active levels of the laser transition in the presence of a standingwave electric field. Equations (7) and (8) follow from Maxwell's equations for the field amplitude of the standing wave in the presence of an atomic or molecular laser medium. Equations (1)-(8) are valid for values of $k$ that are larger than unity. However, for $k=1$, Eq. (3) couples to a more negative value of $k$. In this case, Eq. (3) can be replaced with ${ }^{19}$

$$
\begin{aligned}
\frac{\partial}{\partial t} D_{1}^{\prime}(V, t)= & -h_{1} D_{1}^{\prime}(V, t)-h_{2} M_{1}^{\prime}(V, t) \\
& -2 \gamma_{1}\left[A_{r}(t) P_{i, 1 i}(V, t)\right. \\
& \left.-A_{i}(t) P_{r, 1 i}(V, t)\right],
\end{aligned}
$$

where $P_{r, 1 i}(V, t)$ and $P_{i, 1 i}(V, t)$ are the imaginary parts of the variables $P_{r, 1}(V, t)$ and $P_{i, 1}(V, t)$, respectively. Equations (1)-(8) and (12) form a complete set that uses $k$ values of unity and larger. It is in this form that the equations have been numerically solved for our theoretical investigations of spontaneous coherent pulsations in standing-wave $3.39-\mu \mathrm{m} \mathrm{He}-\mathrm{Ne}$ lasers.

\section{DATA}

In order to apply the model of Section 2 to a specific type of laser, it is necessary to obtain numerical values for the parameters that appear in the model. In this section we use published data to extract values for the parameters of the 3.39- $\mu \mathrm{m} \mathrm{He}-\mathrm{Ne}$ laser.

Among the most fundamental parameters for any laser are the lifetimes of the upper and lower laser levels and the decays between these levels. For the 3.39- $\mu \mathrm{m} \mathrm{He-Ne} \mathrm{laser} \mathrm{transition,} \mathrm{the} \mathrm{upper} \mathrm{and} \mathrm{lower} \mathrm{la-}$ ser levels are identified in Paschen's notation as $3 s_{2}$ and $3 p_{4}$, respectively. The upper level is understood to be subject to the radiation-trapping effect in which the ultraviolet radiation (wavelength $\approx 600.04 \AA$ ) of atomic transitions from the $3 s_{2}$ level to the ground-state level gets reabsorbed by atoms in that state. This causes the effective lifetime of the upper laser state to be longer than its natural lifetime when the trapping effect is not present. For most gas lasers, the trapping is considered to be complete at pressures in excess of a few tens of micrometers (milliTorr). ${ }^{20}$ The values of both the non-radiation-trapped $\Sigma A_{\left(3 s_{2}\right)}$ and the resonance transition $A_{\left(3 s_{2} ; 600.04 \AA\right)}$ decay rates of the $3 s_{2}$ level based on the published data are approximately $46 \times 10^{6} \mathrm{~s}^{-1}$ and $30 \times 10^{6} \mathrm{~s}^{-1}$, respectively. The ratio of the resonance-transition decay rate to the nonradiation-trapped decay rate gives the value of the branching ratio of the resonance transition of $\kappa \approx 0.65$. The total radiation-trapped decay rate of the upper laser level $\Sigma^{\prime} A_{\left(3 s_{2}\right)}$ can be calculated from the formula $\Sigma^{\prime} A_{\left(3 s_{2}\right)}=(1-\kappa) \times \Sigma A_{\left(3 s_{2}\right)}=(1-0.65) \times 46 \times 10^{6}$ $\approx 16 \times 10^{6} \mathrm{~s}^{-1}$. This is the value that we used for the upper laser-level decay rate $\gamma_{a}$ in our calculations. Further details on how to derive these values from the published decay rates of the $3 s_{2}$ level in Refs. 21-24 are available in Ref. 25.

The decay rate of the $3 p_{4}$ level may be calculated from the value of the natural linewidth $\Delta \nu_{n}$ and the decay rate of the $3 s_{2}$ level. It should be noted, however, that the natural linewidth of a transition is determined by the non-radiation-trapped decay rates. ${ }^{20}$ Therefore we used the previously obtained non-radiation-trapped decay rate of the upper level $\Sigma A_{\left(3 s_{2}\right)}$ and adopted the value of $20 \pm 2 \mathrm{MHz}$ for the natural linewidth in our calculation of the $3 p_{4}$ level decay rate. ${ }^{14}$ The result yields the decay rate of the $3 p_{4}$ level of $\gamma_{b}=2 \pi \Delta \nu_{n}-\Sigma A_{\left(3 s_{2}\right)}=2 \pi$ 
$\times 20 \times 10^{6}-46 \times 10^{6} \approx 80 \times 10^{6} \mathrm{~s}^{-1}$. In addition, we used the value of the direct decay rate between the upper and lower laser levels $\gamma_{a b} \approx 2.87 \times 10^{6} \mathrm{~s}^{-1} \cdot{ }^{26}$

The homogeneous linewidth $\Delta \nu_{h}$ has contributions from both the natural linewidth and the pressure broadening. For the broadening coefficients, we use 12.4 $\pm 4.9 \mathrm{MHz} /$ Torr for neon and $25.6 \pm 0.6 \mathrm{MHz} /$ Torr for helium. ${ }^{27}$ In the experiments reported in Ref. 14, the laser was filled with a mixture of single-isotope neon gas and research-grade helium at partial pressures of 150 mTorr and 500 mTorr, respectively. With these values, the pressure-broadening contribution from both gases to the homogeneous linewidth is $12.4 \times 0.15+25.6 \times 0.50$ $\approx 15 \mathrm{MHz}$. The combination of this pressurebroadening of $15 \mathrm{MHz}$ and the natural linewidth of 20 $\mathrm{MHz}$ gives the homogeneous linewidth $\Delta \nu_{h} \approx 35 \mathrm{MHz}$. This in turn yields the polarization decay rate $\gamma=\pi \Delta \nu_{h}$ $\approx 110 \times 10^{6} \mathrm{~s}^{-1}$. An accepted value of the Dopplerbroadening linewidth $\Delta \nu_{D}$ for this lasing transition is approximately $290 \pm 10 \mathrm{MHz} .{ }^{14}$ With these linewidths, the natural damping ratio defined above is $\epsilon \approx 0.10$.

Values are also required for the rate of strong velocitychanging collisions of both the upper and lower laser levels. According to Smith, the total cross-relaxation rate for the upper laser level $\Gamma_{a}$ can be obtained from the relationship ${ }^{28}$

$$
\Gamma_{a}=\Gamma_{a}^{\prime}+x \kappa \sum A_{\left(3 s_{2}\right)},
$$

where $\Gamma_{a}^{\prime}$ and $\kappa$ are the basic collision-rate characteristic of the upper laser level and the branching ratio of the resonance transition from the upper laser level to the ground-state level, respectively. $\sum A_{\left(3 s_{2}\right)}$ is the nonradiation-trapped decay rate of the upper laser level as defined above. The parameter $x$ was defined to be the fraction of active atoms that interacts with the laser beam and absorbs the resonance-transition radiation. This parameter is directly related to the active mode volume inside the cavity. The cavity configuration used in the experiments yields beam spot sizes at the wedge and curved mirror of $0.33 \mathrm{~mm}$ and $0.43 \mathrm{~mm}$, respectively. This results in a mode volume that almost fills the 1-mm innerdiameter capillary tube that was used as the discharge tube. The value $x \approx 1$ can then be used without significant error.

For the pressure at which the experiments were conducted, an interpretation of graphical data reported by $\mathrm{Smith}^{28}$ yields the basic collision rate $\Gamma_{a}^{\prime}$ of approximately $20 \times 10^{6} \mathrm{~s}^{-1}$ for the upper laser level $3 s_{2}$. Equation (13) above then yields the cross-relaxation rate for the upper laser level of $\Gamma_{a}=20 \times 10^{6}+\left(0.65 \times 46 \times 10^{6}\right) \approx 50$ $\times 10^{6} \mathrm{~s}^{-1}$, where the values $x=1, \quad \kappa=0.65$, and $\sum A_{\left(3 s_{2}\right)}=46 \times 10^{6} \mathrm{~s}^{-1}$ have been used. As determined in Ref. 14, we have employed a value for the cavity lifetime of $t_{c}=1 \mathrm{~ns}$, which is equivalent to a decay rate for the electric field of $\gamma_{c}=500 \times 10^{6} \mathrm{~s}^{-1}$.

The uncertainties in the decay rates discussed above are not necessarily negligible. We used these decay rates as a starting point and then iteratively substituted slightly different values into the simulations to see whether any changes within parameter uncertainties
Table 1. Parameters of the 3.39- $\mu \mathrm{m} \mathrm{He-Ne}$ Laser Transition

\begin{tabular}{ll}
\hline \multicolumn{1}{c}{ Parameter } & \multicolumn{1}{c}{ Value } \\
\hline Upper laser-level decay rate, $\gamma_{a}$ & $16 \times 10^{6} \mathrm{~s}^{-1 a}$ \\
Lower laser-level decay rate, $\gamma_{b}$ & $80 \times 10^{6} \mathrm{~s}^{-1}$ \\
Interlevel decay rate, $\gamma_{a b}$ & $2.87 \times 10^{6} \mathrm{~s}^{-1}$ \\
Polarization decay rate, $\gamma$ & $110 \times 10^{6} \mathrm{~s}^{-1}$ \\
Cavity decay rate, $\gamma_{c}$ & $500 \times 10^{6} \mathrm{~s}^{-1}$ \\
Upper laser-level cross-relaxation rate, $\Gamma_{a}$ & $60 \times 10^{6} \mathrm{~s}^{-1}$ \\
Lower laser-level cross-relaxation rate, $\Gamma_{b}$ & $60 \times 10^{6} \mathrm{~s}^{-1}$ \\
Homogeneous linewidth, $\Delta \nu_{h}$ & $35 \mathrm{MHz}$ \\
Doppler-broadened linewidth, $\Delta \nu_{D}$ & $290 \mathrm{MHz}$ \\
Natural-damping ratio, $\epsilon$ & 0.10 \\
\hline
\end{tabular}

${ }^{a}$ Radiation-trapped decay rate.

might improve agreement with the experiments. This procedure suggests slightly larger values of the strong collision rates for the upper and lower laser levels (60 $\times 10^{6} \mathrm{~s}^{-1}$ instead of $50 \times 10^{6} \mathrm{~s}^{-1}$ ). All other values are as derived above. We have expanded the standing-wave field inside the laser cavity into five spatial harmonics and have represented the Doppler-broadened velocity distribution with 100 velocity classes. The laser parameter values used in our calculations are summarized in Table 1 .

\section{RESULTS}

Figures 1 and 2 show a snapshot of the time-series signal and the corresponding homodyne spectrum at the instability threshold, as obtained from our simulations. The theoretical threshold parameter $r_{\text {th }}=1.61$ agrees well with the experimental value $r_{\text {th }} \approx 1$.7. The time-series signal of the laser intensity is nearly sinusoidal, and its corresponding homodyne spectrum has its peak located at $21.1 \times 10^{6} \mathrm{~s}^{-1}$, which agrees with the reported value of $21 \times 10^{6} \mathrm{~s}^{-1} .14$

It may be helpful at this point to give brief descriptions of the concepts of homodyne and heterodyne spectra. A homodyne spectrum is simply the frequency spectrum of the corresponding time-series signal. It can be experimentally observed by detecting the laser output with a square-law detector and feeding the resulting electrical output signal (which is proportional to the square of the laser electric field) from the detector into a spectrum analyzer. Theoretically, a homodyne spectrum is a Fourier transform of the corresponding time-series data. Its frequency components indicate the spacings between the primary spectral components in the corresponding heterodyne spectrum.

Experimentally, a heterodyne spectrum is obtained by mixing the output of the laser under study with the output from a reference laser on a square-law detector, and this can be modeled mathematically. Let $E_{0}(t)$ $=\left[E_{0}^{\prime}(t) \exp \left(-i \omega_{c} t\right)+E_{0}^{\prime *}(t) \exp \left(i \omega_{c} t\right)\right] / 2$ and $E_{r}(t)$ $=\left[E_{r}^{\prime} \exp \left(-i \omega_{r} t\right)+E_{r}^{\prime *} \exp \left(i \omega_{r} t\right)\right] / 2$ represent the electric field of the laser under study and that of the constantamplitude reference laser, respectively. The carrier angular frequency of the laser under study is $\omega_{c}$, and the 
angular frequency of the reference laser is $\omega_{r}$. The mixing at a square-law detector yields an output current proportional to

$$
\begin{aligned}
i(t)= & \overline{\left[E_{0}(t)+E_{r}(t)\right]^{2}} \\
\approx & \frac{1}{2}\left[\left|E_{0}^{\prime}(t)\right|^{2}+\left|E_{r}^{\prime}\right|^{2}\right] \\
& +\frac{1}{2}\left\{E_{0}^{\prime}(t) E_{r}^{\prime *} \exp \left[-i\left(\omega_{c}-\omega_{r}\right) t\right]\right. \\
& \left.+E_{0}^{\prime *}(t) E_{r}^{\prime} \exp \left[i\left(\omega_{c}-\omega_{r}\right) t\right]\right\},
\end{aligned}
$$

where terms with higher-frequency components are omitted due to the limited response of the detector. The bar above $\left|E_{0}(t)+E_{r}(t)\right|^{2}$ indicates a time average over a period long compared with an optical cycle. Equation (14) consists of three components. The first bracket contains two components, which are the dc bias and the signal that corresponds to the homodyne spectrum. The heterodynespectrum component comes from the beating signal between the laser output and the reference signal in the second bracket. This heterodyne spectrum is centered at the difference between the two carrier frequencies, i.e., at $\left|\omega_{c}-\omega_{r}\right|$. Its magnitude is proportional to the amplitude of the reference laser $E_{r}^{\prime}$. A heterodyne spectrum

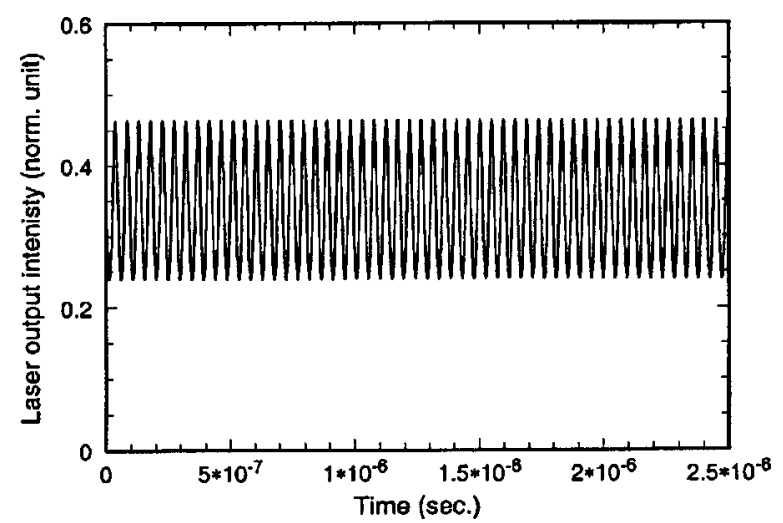

Fig. 1. Time-series signal shows the laser output intensity close to the threshold of instability $\left(r_{\text {th }}=1.61\right)$.

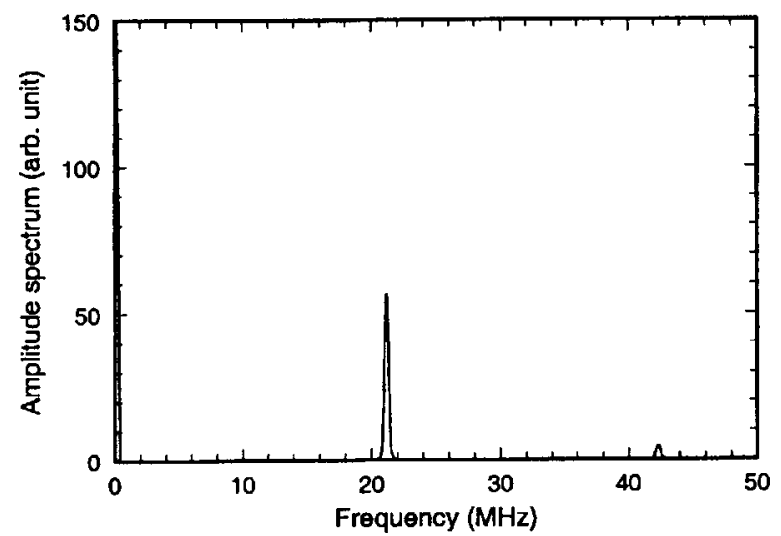

Fig. 2. Homodyne spectrum close to the threshold of instability $\left(r_{\text {th }}=1.61\right)$. provides additional information that is not available in the usual time-series signal or the homodyne spectrum.

The value of the integration step size in our calculations is $0.01 \mathrm{~ns}$. We collect a data point once every 125 integration steps or $125 \times 0.01=1.25 \mathrm{~ns}$. Based on this sampling rate, we have chosen that the reference laser is detuned by $300 \mathrm{MHz}$ above the center of the gain profile of the laser under study, i.e., $\left(\omega_{r}-\omega_{0}\right) /(2 \pi)=300$ $\mathrm{MHz}$, and that the carrier frequency of the laser under study is at the center of the gain profile. This combination yields good representations of the experimental outputs in both time and frequency domains.

Figure 3 shows snapshots of time-series signals together with the corresponding homodyne and heterodyne spectra for five different values of the threshold parameter with line-center tuning. As shown in Fig. 3(a), the heterodyne spectrum of a stable single-mode laser for a threshold parameter $r_{\text {th }}=1.5$ consists of a single peak at the frequency difference between the reference laser and the laser under study. The peak frequency moves with cavity detuning, and this shift corresponds to the shift in the laser operating frequency. The heterodyne spectrum of an unstable laser, however, shows two distinct characteristics that depend on the value of the threshold parameter. At a small value of the threshold parameter, e.g., $r_{\text {th }}=1.66$ as in Fig. 3(b), the spectrum shows a dominant peak with weak sidebands spaced from the peak by amounts that correspond to the frequencies in the associated homodyne spectrum. When the threshold parameter is larger, corresponding to a stronger instability condition, e.g., $r_{\text {th }}=2.6$ in Fig. $3(\mathrm{e})$, the spectrum shows two dominant peaks whose relative magnitudes depend on the amount of cavity detuning. Sideband frequencies are also present, and more complex structures of the frequency spectrum occur at the intermediate values of the threshold parameter represented in Figs. 3(c) and 3(d). This complexity depends on the value of the threshold parameter and on the amount of cavity detuning.

The effects of cavity detuning on the laser outputs are shown in Fig. 4. This figure shows combinations of average laser intensities and peak pulsation frequencies as functions of cavity detuning on the left and laseroperating frequencies as functions of cavity detuning on the right. The peak pulsation frequencies are the frequencies of the principal peaks of the corresponding homodyne spectra. The laser operating frequencies are determined from the heterodyned beat-frequency components in the heterodyne spectra. The curves of average laser intensity versus cavity detuning reveal that the Lamb dip around line center becomes deeper and narrower as the instability-caused pulsing becomes stronger. The dip widens again and gets shallower when the laser is operated further above the lasing threshold, and similar experimental behavior was reported in Fig. 4 of Ref. 14.

The graphs of the laser operating frequencies on the right side of Fig. 4 clearly show increasing deviations from the nearly linear mode-pulling behavior when the threshold parameter increases, as observed also in the experiments. A small kink first develops around the Lambdip region when the threshold parameter is small, and then finally an actual break of the curve occurs. The het- 

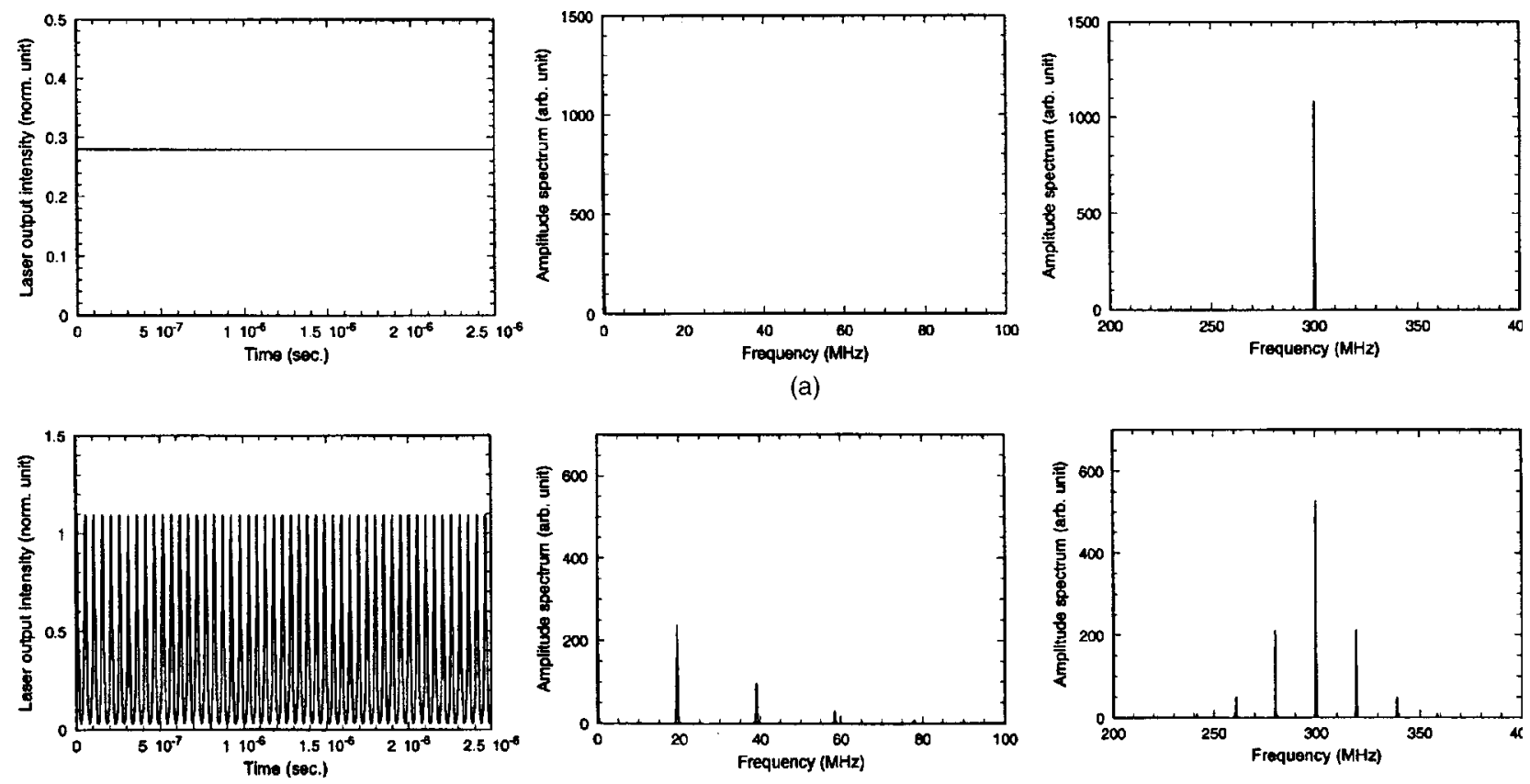

(b)
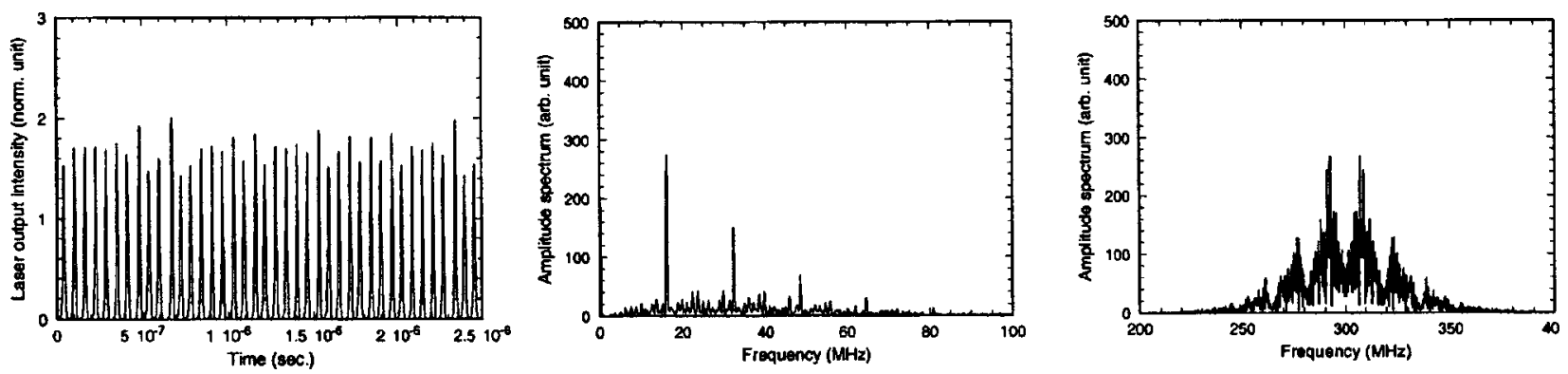

(c)
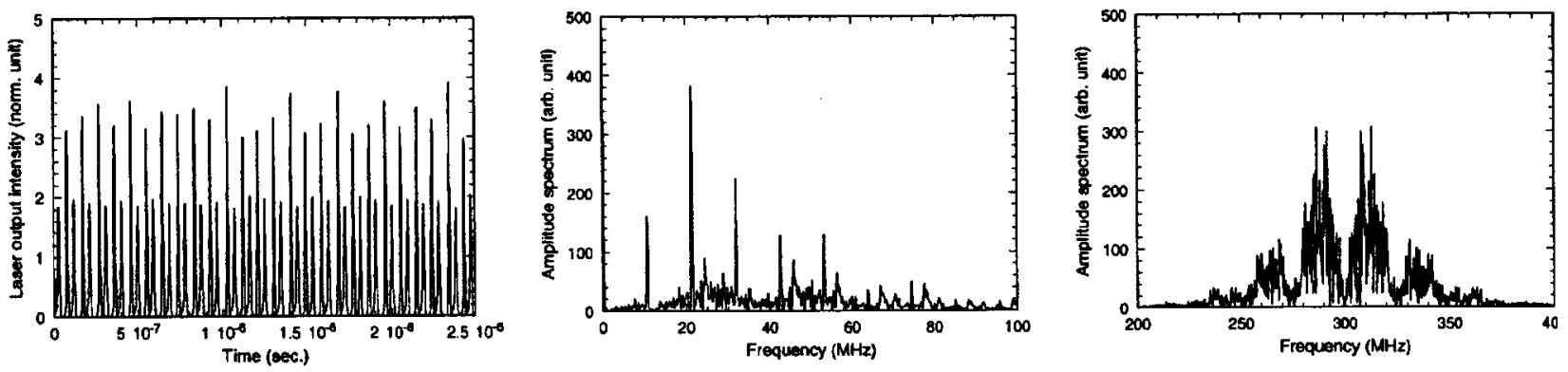

(d)
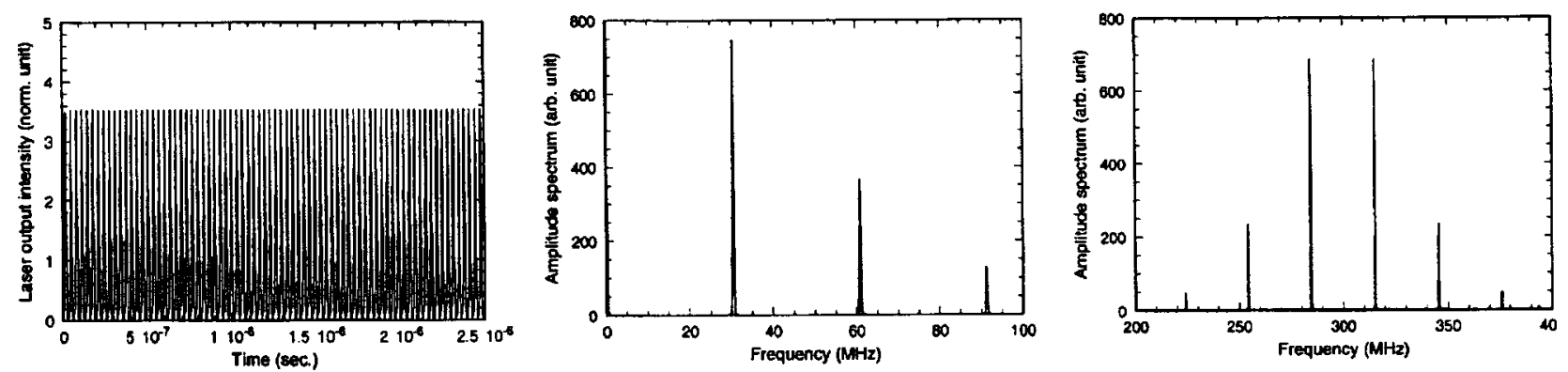

(e)

Fig. 3. Time-series signals show laser output intensities (left). The corresponding homodyne and heterodyne spectra of the observed time-series signals show peak pulsation frequencies and laser operating frequencies (middle and right, respectively). The cavity is tuned to line center, and the values of the threshold parameter are (a) $r_{\text {th }}=1.5$, (b) $r_{\text {th }}=1.66$, (c) $r_{\text {th }}=1.7$, (d) $r_{\text {th }}=2.05$, and (e) $r_{\text {th }}$ $=2.6$. 

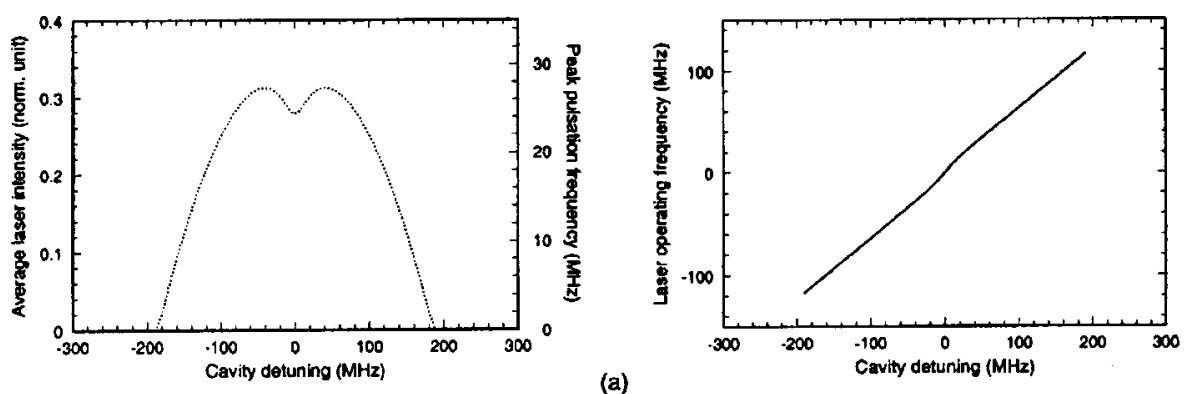

(a)
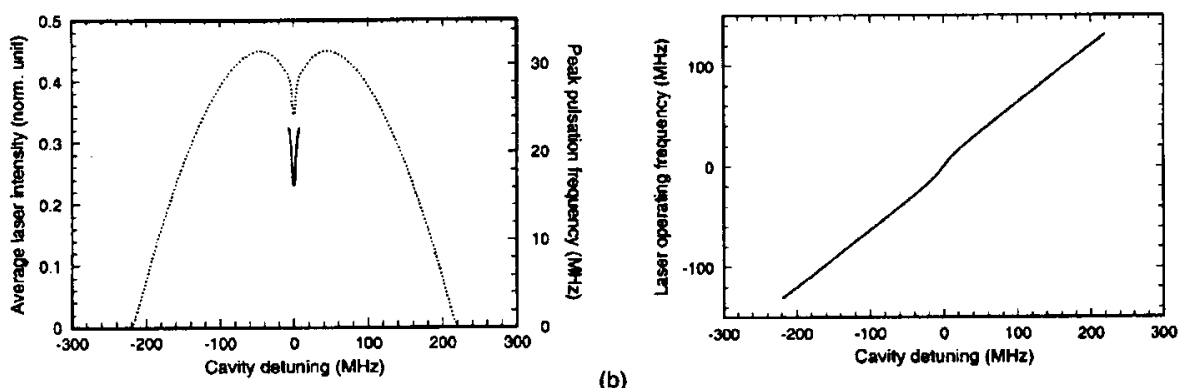

(b)
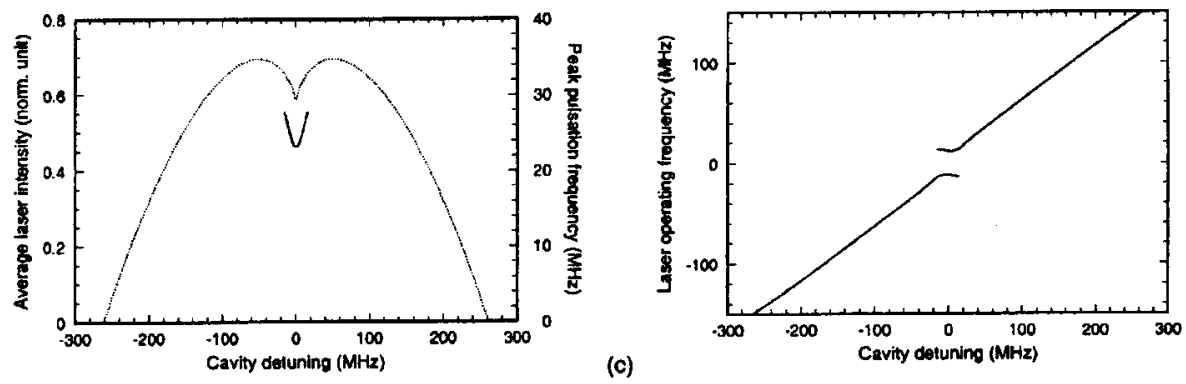

(c)
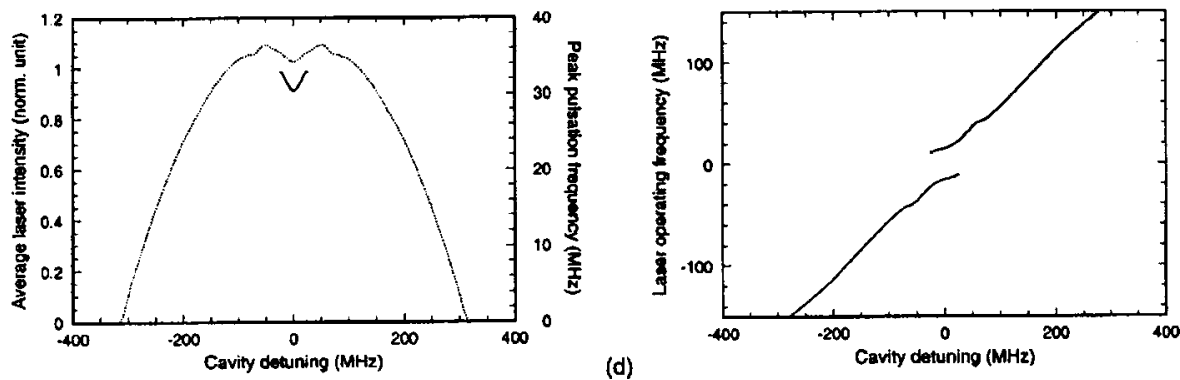

Fig. 4. Average laser intensities (dotted curves) and frequencies of the principal peaks in the homodyne spectra (solid curves) versus cavity detunings (left). Laser operating frequencies versus cavity detunings (right). The threshold parameters are (a) $r_{\text {th }}=1.5$, (b) $r_{\text {th }}=1.7$, (c) $r_{\text {th }}=2.05$, and (d) $r_{\text {th }}=2.6$.

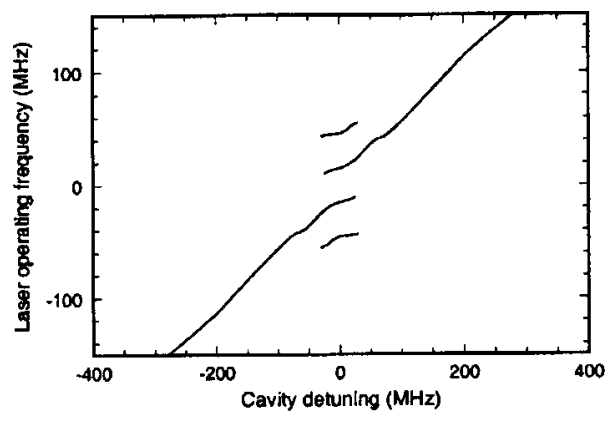

Fig. 5. Expanded scale of Fig. 4(d) showing the details of the laser operating frequency. Note the existence of sidebands that are not displayed in Fig. 4(d). The threshold parameter is $r_{\text {th }}$ $=2.6$. erodyne spectra for the cases when the break in the laseroperating frequency curves occur show two strong beating frequencies, as observed in the experiments and shown in Fig. 4(d), for example. An expanded scale of the laseroperating frequency curves in Fig. 4(d) is shown in Fig. 5. This figure shows additional sideband frequencies that result from the presence of instability.

Figure 6 shows pulsation behaviors at a fixed value of threshold parameter $r_{\text {th }}=2.05$ as a function of cavity detuning. The graphs on the left show the time-series snapshots with the corresponding homodyne spectra on the right. The pulsation behavior changes as the cavity is detuned from line center, with complex pulsations being observed when the laser is operating within a narrow tuning range close to line center. 


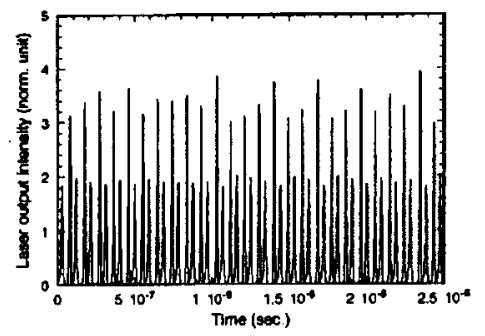

(a)
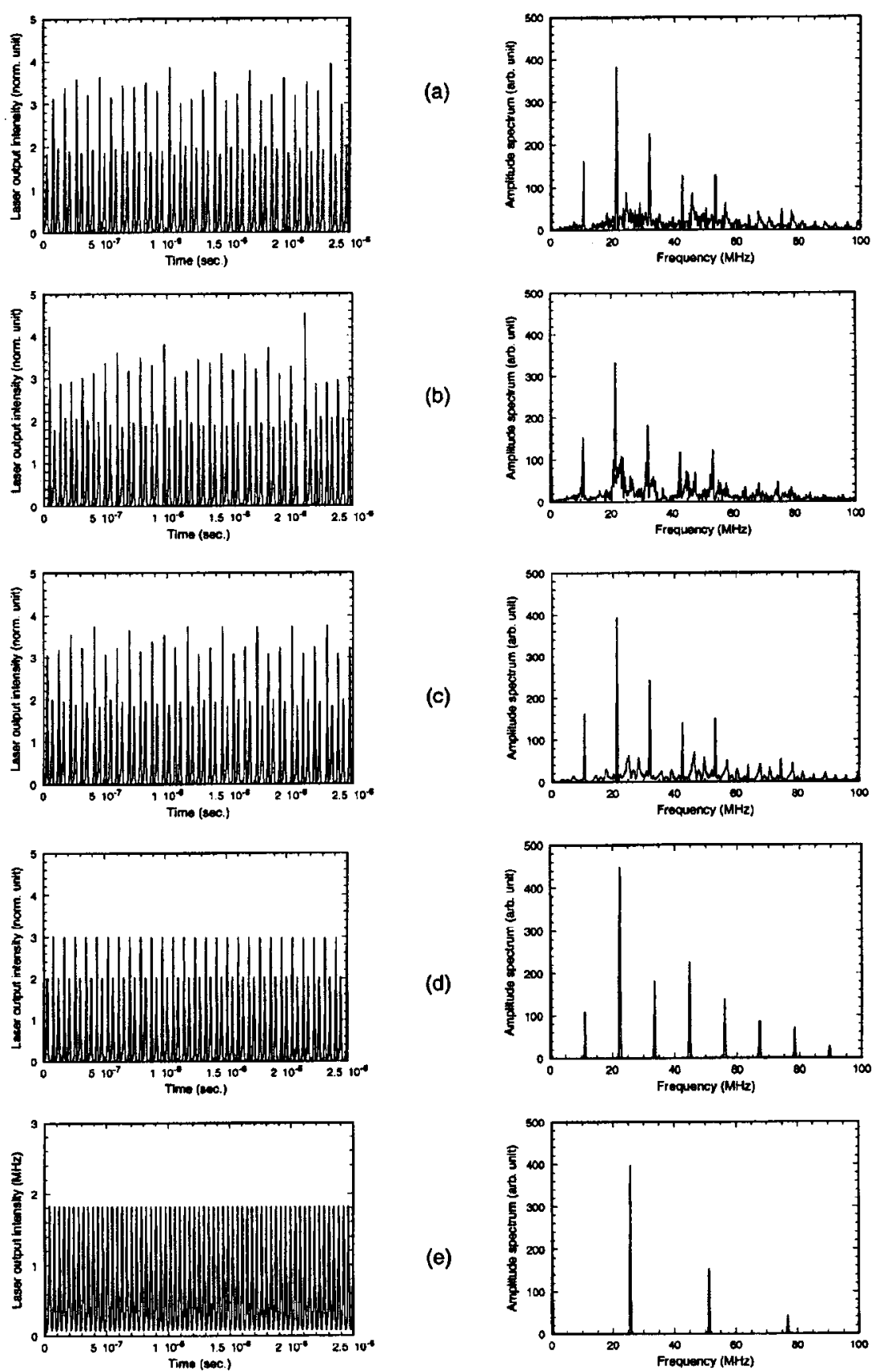

Fig. 6. Laser output intensities (left) and the corresponding homodyne spectra (right) versus cavity detunings. The threshold parameter is $r_{\text {th }}=2.05$. The cavity detunings are (a) line center, (b) $4000 \mathrm{~Hz}$, (c) $0.1 \mathrm{MHz}$, (d) $0.9 \mathrm{MHz}$, and (e) $10 \mathrm{MHz}$.

\section{CONCLUSION}

We have presented detailed theoretical results for the spontaneous coherent pulsations in a single-mode standing-wave 3.39- $\mathrm{m} \mathrm{He}-\mathrm{Ne}$ laser oscillator. These results match both the threshold for instability and the corresponding pulsation frequency in published experimental data. Many behaviors, such as the complex structures of the homodyne and heterodyne spectra as the threshold parameter or the cavity detuning is varied, the narrow range of pulsations around the line center, the anomalous mode pulling, and the varying depth and width of the Lamb dip, are also in agreement with published experimental results.

\section{ACKNOWLEDGMENT}

This research was supported in part by the National Science Foundation under grant ECS-9014481.

\section{REFERENCES}

1. L. W. Casperson and A. Yariv, "The time behavior and spectra of relaxation oscillations in a high-gain laser," IEEE J. Quantum Electron. QE-8, 69-73 (1972).

2. L. W. Casperson, "Spontaneous coherent pulsations in laser oscillators," IEEE J. Quantum Electron. QE-14, 756-761 (1978).

3. L. W. Casperson, "Spontaneous pulsations in lasers," in 
Third New Zealand Symposium on Laser Physics, J. D. Harvey and D. F. Walls, eds., Vol. 182 of Springer Lecture Notes in Physics (Springer-Verlag, Berlin, 1983), pp. 88106 , and references therein.

4. J. R. Ackerhalt, P. W. Milonni, and M.-L. Shih, "Chaos in quantum optics," Phys. Rep. 128, 205-300 (1985).

5. R. G. Harrison and D. J. Biswas, "Pulsating instabilities and chaos in lasers," Prog. Quantum Electron. 10, 147-228 (1985).

6. N. B. Abraham, P. Mandel, and L. M. Narducci, "Dynamical instabilities and pulsations in lasers," in Progress in Optics, E. Wolf, ed. (North-Holland, Amsterdam, 1988), Vol. 25, pp. $1-187$.

7. L. A. Lugiato, L. M. Narducci, J. R. Tredicce, and D. K. Bandy, "25 years of laser instabilities," in Instabilities and Chaos in Quantum Optics II, N. B. Abraham, F. T. Arecchi, and L. A. Lugiato, eds. (Plenum, New York, 1988), pp. 1-25.

8. C. O. Weiss and R. Vilaseca, Dynamics of Lasers (Weinheim, New York, 1991).

9. G. H. M. van Tartwijk and G. P. Agrawal, "Laser instabilities: a modern perspective," Prog. Quantum Electron. 22 43-122 (1998).

10. F. Sanchez and G. Stephan, "General analysis of instabilities in erbium-doped fiber lasers," Phys. Rev. E 53, 2110 2122 (1996)

11. F. Sanchez and A. Kellou, "Laser dynamics with excitedstate absorption,” J. Opt. Soc. Am. B 14, 209-213 (1997).

12. W. Gadomski and B. Ratajska-Gadomska, "Self-pulsations in phonon-assisted lasers," J. Opt. Soc. Am. B 15, 26812688 (1998).

13. R. S. Gioggia and N. B. Abraham, "Single-mode self-pulsing instabilities at the Lamb dip of a He-Ne $3.39 \mu \mathrm{m}$ laser," Opt. Commun. 47, 278-282 (1983).

14. R. S. Gioggia and N. B. Abraham, "Anomalous mode pulling, instabilities, and chaos in a single mode, standingwave $3.39 \mu \mathrm{m}$ He-Ne laser," Phys. Rev. A 29, 1304-1309 (1984).

15. W. E. Lamb, Jr., "Theory of an optical maser," Phys. Rev. 134, A1429-A1450 (1964).
16. L. W. Casperson, "Spontaneous coherent pulsations in ring-laser oscillators," J. Opt. Soc. Am. B 2, 62-72 (1985).

17. M. F. H. Tarroja, N. B. Abraham, D. K. Bandy, and L. M. Narducci, Phys. Rev. A 34, 3148-3158 (1986).

18. L. W. Casperson, "Spontaneous coherent pulsations in standing-wave laser oscillators," J. Opt. Soc. Am. B 5, 958 969 (1988).

19. L. W. Casperson and M. F. H. Tarroja, "Spontaneous coherent pulsations in standing-wave laser oscillators: simplified models," J. Opt. Soc. Am. B 8, 250-261 (1991).

20. W. R. Bennett, Jr., "Gaseous optical masers," Appl. Opt Suppl. 1, 24-61 (1962).

21. B. Decomps and M. Dumont, "T-9 Polarization of the fluorescent light of $\mathrm{Ne}$ atoms interacting with a laser beam measurement of several relaxation times," IEEE J. Quantum Electron. QE-4, 916-922 (1968).

22. G. M. Lawrence and H. S. Liszt, "Radiative lifetimes in the resonance series of Ne," Phys. Rev. 178, 122-125 (1969).

23. J. Z. Klose, "Transition probabilites and mean lives of the $3 s$, laser level in neon I," Phys. Rev. 188, 45-49 (1969).

24. I. P. Konovalov and E. D. Protsenko, "Determination of the relaxation constants of levels by the method of resonant interaction of two travelling waves with a two-level system," Sov. J. Quantum Electron. 6, 1084-1091 (1976).

25. P. Chenkosol, "Spontaneous pulsations in laser oscillators: effects of spatial field distributions; self-modelocking dynamics; pulsations in $3.39 \mu \mathrm{m} \mathrm{He}-\mathrm{Ne}$ standingwave lasers," Ph.D. dissertation (Portland State University, Portland, Ore., 2003).

26. J. T. Verdeyen, in Lasers, 2nd ed. (Prentice-Hall, Englewood Cliffs, N.J., 1989), p. 328.

27. M. Ohi, "Measurements of frequency shifts in the $3.39 \mu \mathrm{m}$ Ne transition with the aid of $\mathrm{CH}_{4}$ Lamb dip," Jpn. J. Appl. Phys. 12, 1377-1381 (1973).

28. P. W. Smith, "The effect of cross relaxation on the behavior of gas laser oscillators," IEEE J. Quantum Electron. QE-8, 704-710 (1972). 